\title{
Special issue on cyber-physical systems and intelligent control in honor of the 65th birthday of Professor Bijoy K. Ghosh
}

\author{
Jiangping $\mathrm{Hu}^{1} \cdot$ Wenxue Wang ${ }^{2}$
}

Received: 24 September 2021 / Accepted: 25 September 2021

(c) The Author(s), under exclusive licence to South China University of Technology and Academy of Mathematics and Systems Science, Chinese Academy of Sciences 2021

It is our great pleasure to organize this special issue in Control Theory and Technology in honor of the 65th birthday of Professor Bijoy K. Ghosh, who has made many truly outstanding contributions to the field of systems and control through the years, which include robust and nonlinear control, robotics and machine vision in his earlier research period, and his recent focus on biology and biomedical modeling, learning control and multi-agent systems to name a few.

Professor Ghosh obtained his Bachelor's degree and Master's degree in Electrical Engineering at Birla Institute of Technology and Science, Pilani, India, in 1977, and at Indian Institute of Technology, Kanpur, India, in 1979, respectively. In 1983, he obtained his Ph.D. in Engineering at Harvard University. Then, he worked at Washington University in Saint Louis for 23 years. In 2007, he became a Regent Professor of Mathematics and Statistics at Texas Tech University, Lubbock, Texas. In 2018, he joined the School of Automation Engineering, University of Electronic Science and Technology of China, Chengdu, China. In the early research period, he followed Professors Roger W. Brockett and Y. C. Ho and interacted with other control theorists, such as Prof. H. Kimura, Profs. M. Hazewinkel and J. C. Willems, Profs. K. S. Narendra and Steve Morse, Prof. Clyde Martin and Prof. H. F. Chen. He applied his research to Aerospace Control, while interacting with engineers in McDonell Douglas Aerospace Company in St. Louis, USA. This research

Jiangping $\mathrm{Hu}$

hujp@uestc.edu.cn

Wenxue Wang

wangwenxue@sia.cn

1 School of Automation Engineering, University of Electronic Science and Technology of China (UESTC), Chengdu 610054, Sichuan, China

2 Shenyang Institute of Automation, Chinese Academy of Sciences, Beijing 100190, China culminated the Donald P. Eckmann Award in 1988 with citation "in recognition of his outstanding contributions in the field of Automatic Control". Starting from 1990, he started working in the field of Robotics and Machine Vision. As an active member of the IEEE, he established the Technical Committee on Sensor Guided Manipulation in Automation (SGMA). In 2000, he became a Fellow of the IEEE with citation "for fundamental contributions to system theory with applications to robust control, vision and multisensory fusion". During 2001-2006, he established the IEEE Technical Committee on Bio Systems and Control. He became a Fellow of IFAC in 2014 with citation "for seminal contributions to dynamic modeling in machine vision, biology and biomedical science".

The key contributions of each manuscript included in this special issue are summarized as follows:

(1) A minimum adequate set of multi-valued logic by Daizhan Cheng, Jun-e Feng, Jianli Zhao, Shihua Fu

The $k$-valued logic theory may play more important role in the design of temporal logic as well as the cyber-physical systems. Some fundamental issues in investigating $k$-valued logic involve the construction of maximum closed sets and minimum adequate sets. This paper provides an adequate set of $k$-valued logic, which contains of only two operators. It is also proved that this adequate set is of minimum size.

(2) Output digitization of simple measure-preserving linear systems by Alisa DeStefano, Makhin Thitsa, Clyde Martin

This paper examines three simple linear systems from the viewpoint of ergodic theory. The output is digitized and only the sign of the output is recorded at integer times. It is shown that even with this minimal output the important information about the systems can be recovered. In particular, the rate of rotation can be determined for a two-dimensional system viewed as a flow on the circle. These results are then used to 
determine the slope of the trajectories for constant irrational flow on the two-dimensional torus. Two four-dimensional systems are examined and reduced for the study of linear flows on the two-dimensional torus.

(3) The dorsolateral pre-frontal cortex bipolar errorrelated potential in a locked-in patient implanted with a daily use brain-computer interface by Zachary Freudenburg, Khaterah Kohneshin, Erik Aarnoutse, Mariska Vansteensel, Mariana Branco, Sacha Leinders, Max van den Boom, Elmar G. M. Pels, Nick Ramsey

This paper investigates the brain signals of an implanted BCI user suffering from locked in syndrome (LIS) due to late-stage ALS that prevents her from being able to speak or move but not from using her BCI at home on a daily basis to communicate, for the presence of error related signals. This paper first establishes the presence of an ErrP originating from the dorsolateral pre-frontal cortex (dLPFC) in response to errors made during a discrete feedback task that mimics the click-based spelling software she uses to communicate. Then, this paper shows that this ErrP can also be elicited by cursor movement errors in a continuous BCI cursor control task. This work represents a first step towards detecting ErrPs during the daily home use of a communications BCI.

(4) Quantum-enhanced reinforcement learning for control: a preliminary study by Yazhou Hu, Fengzhen Tang, Jun Chen, Wenxue Wang

This paper studies a quantum-enhanced reinforcement learning for optimal control, where the states and actions of reinforcement learning are quantized by quantum technology. A probability amplification method is presented to effectively avoid the trade-off between exploration and exploitation via quantized technology. An optimal control policy is learnt during the process of reinforcement learning. The performance of this quantized algorithm is demonstrated in both MountainCar and CartPole reinforcement learning environments. The preliminary study results validate that, compared with Q-learning, this quantized reinforcement learning method has better control performance without taking the trade-off between exploration and exploitation into consideration.

(5) Optimal energy consuming planning for a home-based microgrid with mobility constraint of electric vehicles and tractors by Shota Inuzuka, Tielong Shen

The electric vehicles (EVs) and electric tractors (ETs) function as batteries in the power system, while they often have to leave it for their mobility and agricultural work. This paper deals with the energy management problem to minimize the cost of non- renewable energy for a small-scale microgrid with EVs and ETs. This paper deals with uncertainties by constraints for robust energy planning and expected optimization based on scenarios, and shows that the scheduling of the SoC and the optimal home-based power consumption planning can be obtained.

(6) Support optimal scheduling with weighted random forest for operation resources by $\mathrm{Li} \mathrm{Li}$, Qingyun $\mathrm{Yu}$, Haoyi Shi, Yuguang Liu

Operation-related resources are lots of manpower and material with the characteristics of high cost and high income in hospitals, and scheduling optimization is a very important research issue in medical service. This paper proposes a systematic approach to optimize scheduling problems based on multiple characteristics of operating resources to cope with the actualities of operation resources scheduling. A framework that includes the composite dispatching rules (CDR), optimization ideology, and feedback mechanism, is designed. The optimization ideology is carried out through a learning model based on the weighted random forest (WRF) algorithm.

(7) A solution strategy for distributed uncertain economic dispatch problems via scenario theory by Peng Li, Jiangping $\mathrm{Hu}$

This paper considers an uncertain economic dispatch problem (EDP) for a group of cooperative agents. First, let each agent extract a set of samples (scenarios) from the uncertain set, and then a scenario EDP is followed using these scenarios. Based on the scenario theory, a prior certification is provided to evaluate the probability feasibility of the scenario solution for uncertain EDP. To facilitate the computational task, a distributed solution strategy is proposed using the alternating direction method of multipliers (ADMM) and a finite-time consensus algorithm. Moreover, the distributed optimization strategy can solve the scenario problem over a weight-balanced directed network.

(8) Distributed solver for linear matrix inequalities: An optimization perspective by Weijian Li, Wen Deng, Xianlin Zeng, Yiguang Hong

This paper develops a distributed solver for a group of strict (nonstrict) linear matrix inequalities (LMIs) over a multi-agent network, where each agent only knows one inequality, and all agents cooperate to reach a consensus solution in the intersection of all the feasible regions. The formulation is transformed into a distributed optimization problem by introducing slack variables and consensus constraints. Then, a distributed algorithm is proposed with the help of the primal-dual method, projection operators and derivative feedback. Finally, the convergence of the 
algorithm is analyzed theoretically and illustrated with simulations.

(9) Brain-computer interfaces for human gait restoration by Zoran Nenadic

This review article presents more than a decade of the author's work on the development of brain-computer interface (BCI) systems for the restoration of walking following neurological injuries, such as spinal cord injury (SCI) or stroke. Most of this work has been in the domain of non-invasive electroencephalogram-based BCIs, including interfacing our system with a virtual reality environment and physical prostheses. Extensions of this work include the development of a portable low-cost BCI suitable for at-home use, the ongoing efforts to develop a fully implantable $\mathrm{BCI}$ for the restoration of walking and leg sensation after SCI, and the novel BCI-based therapy for stroke rehabilitation.

(10) Sequencing of multi-robot behaviors using reinforcement learning by Pietro Pierpaoli, Thinh T. Doan, Justin Romberg, Magnus Egerstedt

Given a collection of parameterized multi-robot controllers associated with individual behaviors designed for particular tasks, this paper considers the problem of how to sequence and instantiate the behaviors for the purpose of completing a more complex, overarching mission. In this paper, this problem is dealt with using reinforcement learning to approximate the solution to the computationally intractable sequencing problem, combined with an online gradient descent approach to selecting the individual behavior parameters, while the transitions among behaviors are triggered automatically when the behaviors have reached a desired performance level relative to a task performance cost. To illustrate the effectiveness of the proposed method, it is implemented on a team of differential-drive robots for convoy protection and object manipulation.

(11) Improving the classification accuracy using biomarkers selected from machine learning methods by Linduni M. Rodrigo, Ashoka D. Polpitiya

High-dimensional data encountered in genomic and proteomic studies are often limited by the sample size but has a higher number of predictor variables. Therefore, selecting the most relevant variables that are correlated with the outcome variable is a crucial step. This paper describes an approach for selecting a set of optimal variables to achieve a classification model with high predictive accuracy. The work described using a biological classifier published elsewhere but it can be generalized for any application.

(12) Control engineering of continuous-mode single-photon states: A review by Guofeng Zhang

Recently, there has been a rapidly growing interest in the generation, communication, storage, and manipulation (e.g., pulse shaping) of few-photon states. Thus, a new and important problem in the field of quantum engineering is: How to analyze and design quantum dynamical systems driven by fewphoton states so as to achieve desirable control performance? This survey article presents single-photon states of electromagnetic fields, discusses discrete measurements of a single-photon field, shows how a linear quantum system responds to a single-photon input, investigates how a coherent feedback network can be used to manipulate the temporal pulse shape of a single-photon state, presents single-photon filter and master equations, and finally discusses the generation of Schrödinger cat states by means of photon addition and subtraction.

(13) Discrete-time inverse linear quadratic optimal control over finite time-horizon under noisy output measurements by Han Zhang, Yibei Li, Xiaoming $\mathrm{Hu}$

This paper considers a problem of inverse quadratic optimal control over finite time-horizon for discretetime linear systems. The goal is to recover the corresponding quadratic objective function using noisy observations. First, the identifiability of the model structure for the inverse optimal control problem is analyzed under relative degree assumption. Next, the inverse optimal control problem is studied under the cases with unknown initial state distribution and the observation noise distribution. This paper also studies the case where the exact observations on the initial states are not available, yet the observation noises are known to be white Gaussian distributed and the distribution of the initial state is also Gaussian (with unknown mean and covariance).

Guest editors:

Jiangping $\mathrm{Hu}$

Wenxue Wang 


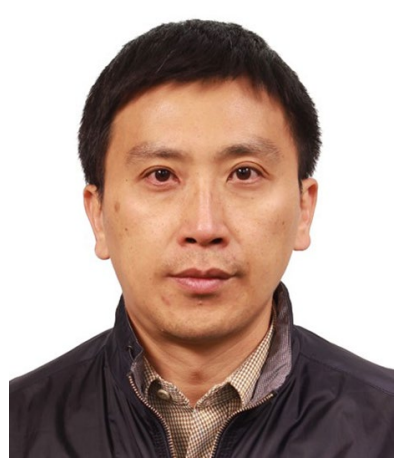

Jiangping $\mathrm{Hu}$ received the B.Sc. degree in applied mathematics and the M.Sc. degree in computational mathematics from Lanzhou University, China, in 2000 and 2004, respectively. He received the $\mathrm{Ph} . \mathrm{D}$. degree in modelling and control of complex systems from Academy of Mathematics and Systems Science (AMSS), Chinese Academy of Sciences (CAS), Beijing, China, in 2007. He has held various positions at Royal Institute of Technology (KTH), Sweden, City University of Hong Kong, Hong Kong, China, Sophia University, Japan, University of Western Sydney, Australia. Currently he is a professor at the School of Automation Engineering, University of Electronic Science and Technology of China (UESTC), Chengdu, China. His current research interests include multi-agent systems, social dynamics and sensor networks. Dr. $\mathrm{Hu}$ has served as an associate editor of the journal KYBERNETIKA and an associate editor of the Journal of Systems Science and Complexity.

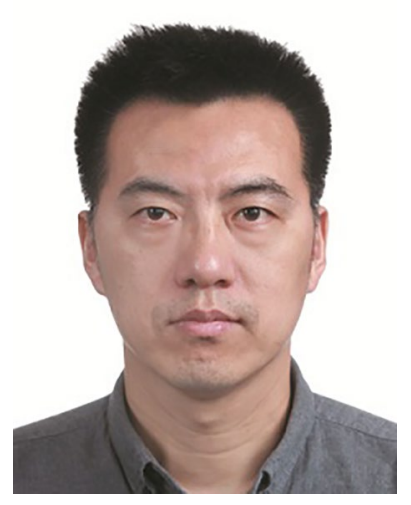

Wenxue Wang received the B.Sc. degree in automatic control from the Beijing Institute of Technology, Beijing, China, the M.Sc. degree in control theory from the Institute of Systems Science, Chinese Academy of Sciences, Beijing, and the D.Sc. degree in systems science and mathematics from Washington University in Saint Louis, St. Louis, MO, USA, in 1996, 1999, and 2006, respectively. He is currently a Professor at Shenyang Institute of Automation, Chinese Academy of Sciences. His research interests include machine learning, compressive sensing, microbotics, biosyncretic robot, and computational and systems biology. 